Como citar este artigo: MARCHEZAN, NATHANY C.; MAIER, PAOLA M.; BASSI, BIANCA C. C; MACHADO, MITHIELLE A.; PONTE. ALINE S.

O desafio da interprofissionalidade: percepção dos profissionais de Fisioterapia e Terapia Ocupacional sobre a atuação em equipe no Rio Grande do Sul. Revista Saúde (Sta. Maria). 2020; 46 (2).

\section{Autor correspondente:}

Nome: Nathany Caleiro Marchezan

E-mail: nathanymarchezan@hotmail.com Telefone: (055) 98444- 1362

Formação Profissional: Terapeuta Ocupacional pela Universidade Federal de Santa Maria (UFSM), Santa Maria, RS, Brasil.

Filiação Institucional: Universidade Federal de Santa Maria

Endereço para correspondência:

Av. Roraima, $n^{\circ}$ 1000, prédio 26D

Bairro: Camobi

Cidade: Santa Maria

Estado: Rio Grande do Sul

CEP: 97 105-900.

Data de Submissão:

24/08/2019

Data de aceite:

10/08/2020

Conflito de Interesse: Não há conflito de interesse

\section{(cc) $\mathrm{BY}-\mathrm{NC}-\mathrm{ND}$}

\title{
O desafio da interprofissionalidade: percepção dos profissionais de Fisioterapia e Terapia Ocupacional sobre a atuação em equipe no Rio Grande do Sul
}

\section{The challenge of interprofessionality: perception of Physical Therapy and Occupational Therapy professionals on teamwork in Rio Grande do Sul}

\author{
Nathany Caleiro Marchezan, Paola Machado Maier, \\ Bianca Gonçalves de Carrasco Bassi, Mithielle de Araújo Machado, \\ Aline Sarturi Ponte
}

\section{RESUMO}

Introdução: Atualmente há grande relevância de compreender a percepção sobre atuação em equipe entre os profissionais de Fisioterapia e Terapia Ocupacional. Objetivo: Este estudo tem por objetivo compreender a percepção dos profissionais de Fisioterapia e Terapia Ocupacional quanto à atuação em equipe. Método: Descritivo, exploratório, metodologia mista - quali-quantitativa. A coleta de dados ocorreu nos meses de maio e junho de 2018. Os dados quantitativos foram analisados descritivamente pelo software Statistica 9.1 e os qualitativos foram analisados pela Análise de Conteúdo. Resultados: Participaram deste estudo 161 profissionais formados, sendo 131 (81,4\%) Fisioterapeutas e 30 (18,6\%) Terapeutas Ocupacionais, credenciados ao Conselho Regional de Fisioterapia e Terapia Ocupacional - CREFITO-5. Quanto ao sexo pode-se observar a predominância de profissionais do sexo feminino (142 [88,2\%]), a média de idade foi de 34,03 anos, a área de atuação profissional que prevaleceu foi a Neurologia (17 [11,1\%]) seguida da Geral $(16[9,5 \%])$, a maioria dos profissionais trabalha em hospitais $(23[11,6 \%])$, todavia não foi o único local, a maioria dos participantes atua em equipe multiprofissional $(53,1 \%)$ e estão concentrados na região metropolitana do Estado do Rio Grande do Sul (62 [42,3\%]). Considerações Finais: através desse estudo, observou-se a dificuldade de compreensão por parte dos profissionais em relação aos termos multiprofissional e interprofissional e de como aplicá-los na prática de atuação em equipe. Há necessidade de um ensino em saúde que propicie a formação de profissionais comprometidos com a integralidade, atores ativos no processo de melhoria da resolutividade do sistema de saúde.

PALAVRAS-CHAVE: Terapia Ocupacional; Fisioterapia; Equipe de assistência ao paciente.

\section{ABSTRACT}

Introduction: Currently there is great relevance to understand the perception about team performance among professionals in Physical Therapy and Occupational Therapy. Objective: This study aims to understand the perception of professionals in Physical Therapy and Occupational Therapy regarding team performance. Method: Descriptive, exploratory, mixed methodology - qualitative and quantitative. Data collection was performed in May and June 2016. Quantitative data were analyzed descriptively by Statistica 9.1 software and qualitative data were analyzed by Content Analysis. Results: 161 professionals participated in this study, of which $131(81.4 \%)$ were Physical Therapists and 30 (18.6\%) were Occupational Therapists accredited to the Regional Council of Physical Therapy and Occupational Therapy - CREFITO-5. As for sex, the predominance of female professionals (142 [88.2\%]), the mean age was 34.03 years, the area of professional activity that prevailed was Neurology $(17[11,1 \%])$ followed by General (16 [9.5\%]), most professionals work in hospitals (23 [11.6\%]), however it was not the only place, most of the participants work in a multiprofessional team $(53,1 \%)$ and are concentrated in the metropolitan region of the State of Rio Grande do Sul (62 [42.3\%]). Final Considerations: through this study, it was observed the difficulty of understanding by professionals in relation to the multiprofessional and interprofessional terms and how to apply them in the practice of teamwork. There is a need for health education that fosters the formation of professionals committed to integrality, active actors in the process of improving the health system's resolution. 


\section{INTRODUÇÃO}

Este estudo foi desenvolvido com o intuito de conhecer a realidade sobre a atuação em equipe entre os profissionais de Fisioterapia e Terapia Ocupacional no estado Rio Grande do Sul, buscando compreender como ocorrem estas atuações em equipe nos serviços de saúde.

Em 1946 a Organização Mundial da Saúde define saúde como, "o completo estado de bem-estar físico, mental e social e não apenas a ausência de doença ou enfermidade $(s / p)^{1}$. Esta conceituação de saúde aparentemente inacessível, apresenta uma estreita relação com as condições de desenvolvimento humano e social, associando à saúde a qualidade de vida das populações. Sendo assim, os determinantes não são somente biológicos e genéticos, estes também são sociais, econômicos e ambientais ${ }^{2}$. Assim, a saúde constitui-se de uma produção social, cultural, familiares, crenças e experiências políticas, influenciada pelas condições de vida, são elas as dimensões social, biológica e ético-política².

A busca por um cuidado integral, que não contemple somente os processos de adoecimento, mas todas as dimensões da vida exigem dos serviços de saúde a constituição de equipes de atenção à saúde, pois "uma especialidade não conseguiria cuidar de todos os âmbitos da vida isoladamentenecessitando da atenção de mais de um profissional para garantir o cuidado integral [...]" (p.148)3.

A atuação em equipe é uma estratégia de atenção em saúde cada vez mais utilizada nos serviços de saúde, esta também é uma proposta oficial do Ministério da Saúde e Educação ${ }^{4}$. As equipes são um conjunto de pessoas que têm uma vinculação entre si, para a realização de tarefas/trabalhos compartilhados e em coletivamente alcançam os objetivos comuns pretendidos ${ }^{5}$. Estas caracterizam-se como organizações mais criativas e eficientes na resolução de problemas, produzem mais e com maior qualidade, desenvolvem maior autonomia e são mais motivadas ${ }^{6}$.

As equipes de atenção à saúde multiprofissionais são constituídas por profissionais de diferentes áreas, que não necessariamente atuam em conjunto. 0 trabalho da equipe multiprofissional é

uma modalidade de trabalho coletivo que se configura na relação recíproca entre múltiplas intervenções técnicas e a interação dos agentes de diferentes áreas profissionais. Por meio da comunicação, ou seja, da mediação simbólica da linguagem, dá-se a articulação das ações multiprofissionais e a cooperação ${ }^{7}$ (p.108).

As práticas das equipes interprofissionais estão relacionadas a forma como se desenvolve e articula as ações entre os profissionais. Nestas equipes os profissionais realizam um trabalho integrado e colaborativo que agregam na atuação da equipe, ampliando a compreensão e trocas de saberes, seguindo os moldes da interdisciplinaridade. Nesta equipe o trabalho é

marcado pela reflexão sobre os papéis profissionais, a resolução de problemas e a negociação nos processos decisórios, a partir da construção de conhecimentos, de forma dialógica e com respeito às singularidades e diferenças dos diversos núcleos de saberes e práticas profissionais ${ }^{8}$ (p.602) 
Os conceitos apresentados têm se tornado parte da prática cotidiana dos profissionais de saúde, nos mais diversos campos de atuação e serviços de atenção à saúde. Sendo assim, torna-se relevante compreender a percepção dos profissionais de Fisioterapia e Terapia Ocupacional quanto à atuação em equipe.

\section{MÉTODO}

Este estudo caracteriza-se como descritivo, misto (quali-quantitativa). Este foi realizado com profissionais de Fisioterapia e Terapia Ocupacional credenciados ao Conselho Regional de Fisioterapia e Terapia Ocupacional da $5^{a}$ Região - CREFITO-5, RS. Obteve aprovação do Comitê de Ética em Pesquisa sob parecer número 2.592.390.

Para a coleta de dados as pesquisadoras contaram com o apoio do CREFITO-5. Esta ocorreu através de um questionário eletrônico organizado na plataforma virtual - Google Forms, que foi encaminhando via e-mail aos profissionais credenciados no CREFITO-5. O questionário era composto por 10 questões, sendo 04 questões fechadas (sexo, profissão, se no local de trabalho atua em equipe e se esta caracteriza-se como interprofissional ou multiprofissional) e 06 abertas (idade, região do estado que atua, instituição que trabalha, tempo de atuação na profissão, área de atuação e a percepção do profissional sobre a atuação em equipe). Ao receber os participantes acessavam primeiramente o Termo de Consentimento Livre e Esclarecido - TCLE, ao final do documento tinham um espaço para assinalar as opções "concordo em participar" ou "não concordo em participar", ao concordar o participante era remetido diretamente ao questionário, caso não concordassem a página era encerrada. A coleta de dados ocorreu nos meses de maio e a junho de 2018.

Para a análise dos dados qualitativos utilizou-se a Análise de Conteúdo ${ }^{9}$. E os quantitativos foram analisados através de análise descritiva com o auxílio do Software Statistica9 ${ }^{9}$.

\section{RESULTADOS E DISCUSSÕES}

Os dados estão organizados em três categorias, são elas: i) caracterização dos profissionais de Fisioterapia e Terapia Ocupacional; ii) atuação em equipe e; iii) atuação em equipe e o sujeito.

\section{Categoria 1 - Caracterização dos profissionais de Fisioterapia e Terapia Ocupacional}

Em maio de 2018 o CREFITO-5 contava com 13.397 Fisioterapeutas e 872 Terapeutas Ocupacionais, totalizando 14.269 profissionais credenciados10, este total de profissionais foi convidado a participar deste estudo. Aderiram 161 profissionais, sendo 131 (81,4\%) Fisioterapeutas e 30 (18,6\%) Terapeutas Ocupacionais. A diferença do número de profissionais entre as profissões pode ser justificado pelo número cursos superior na região Sul do Brasil (Paraná, Santa Catarina e Rio Grande do Sul), segundo um estudo de Silva ${ }^{11}$, esta região conta com 10 cursos de Terapia Ocupacional e 85 cursos de Fisioterapia, cadastrados no e-MEC. 
Observou-se a prevalência do sexo feminino (Tabela 1), sendo esta comum em cursos da área da saúde também como demonstram outros estudos ${ }^{12,13}$. A média de idade 34,03 anos (mínima 22 e máxima 58 anos), não se encontrou nas bases científicas consultadas estudo na literatura nacional e internacional que discutisse este dado.

Tabela 1 - Caracterização dos Profissionais ( $n=161)$.

\begin{tabular}{|c|c|c|}
\hline Variável & Frequência (n) & Percentual (\%) \\
\hline \multicolumn{3}{|l|}{ Profissão } \\
\hline Fisioterapeutas & 131 & $81,4 \%$ \\
\hline Terapeutas Ocupacionais & 30 & $18,6 \%$ \\
\hline \multicolumn{3}{|l|}{ Sexo } \\
\hline Feminino & 142 & $88,2 \%$ \\
\hline Masculino & 19 & $11,8 \%$ \\
\hline
\end{tabular}

Fonte: elaborada pelas autoras.

Das áreas de atuação apresentadas na Tabela 2, pode-se observar que a maioria dos participantes não especificaram a área de atuação. Em relação aos serviços, observa-se que a maioria dos participantes atuam em hospitais (Tabela 2). A média de tempo de trabalho foi de 9,4 anos (mínimo um ano e máximo 33 anos).

Tabela 2 - Apresentação das áreas e serviços que atuam ( $n=161)$.

\begin{tabular}{|c|c|c|}
\hline Variável & Frequência (n) & Percentual (\%) \\
\hline \multicolumn{3}{|l|}{ Área } \\
\hline Não especificaram a área de atuação & 40 & $17,5 \%$ \\
\hline Neurologia & 17 & $11,1 \%$ \\
\hline Geral & 16 & $9,5 \%$ \\
\hline Ortopedia e Traumatologia & 14 & $9,4 \%$ \\
\hline Traumatologia, Ortopedia, Neurologia & 9 & $6,4 \%$ \\
\hline Hospitalar & 8 & $5,9 \%$ \\
\hline Saúde Mental & 8 & $5,9 \%$ \\
\hline Geriatria e Gerontologia & 7 & $4,5 \%$ \\
\hline Docência e Planejamento em saúde & 5 & $2,7 \%$ \\
\hline Ergonomia e saúde do trabalhador & 5 & $2,7 \%$ \\
\hline Acupuntura & 3 & $2,1 \%$ \\
\hline Cardiorrespiratória & 3 & $2,1 \%$ \\
\hline Pediatria & 3 & $2,1 \%$ \\
\hline Pilates e estética & 3 & $2,1 \%$ \\
\hline Reabilitação intelectual, física e autismo & 3 & $2,1 \%$ \\
\hline Saúde Pública & 3 & $2,1 \%$ \\
\hline Terapia intensiva & 3 & $2,1 \%$ \\
\hline Outros & 11 & $9,7 \%$ \\
\hline
\end{tabular}




\section{Serviços}

\begin{tabular}{lll} 
Hospital & 23 & $11,6 \%$ \\
Autônomo & 20 & $10,4 \%$ \\
Clínica & 20 & $10,4 \%$ \\
Não especificou & 20 & $10,4 \%$ \\
Domiciliar & 14 & $9,2 \%$ \\
Prefeitura Municipal & 11 & $8,1 \%$ \\
Consultório & 10 & $7,2 \%$ \\
Universidade privada & 10 & $7,2 \%$ \\
Associação Pais e Amigos dos & & \\
Excepcionais - APAE & 9 & $6,3 \%$ \\
Universidade pública & 9 & $6,3 \%$ \\
Empresa privada & 3 & $2,1 \%$ \\
Secretária Estadual de Saúde & 3 & $2,1 \%$ \\
Outros & 9 & $8,7 \%$ \\
\hline
\end{tabular}

Fonte: elaborada pelas autoras.

Pode-se observar que a maioria dos participantes estão concentrados na região metropolitana do Estado (Tabela 3). Esta região do Estado conta com um total de 5.292 Fisioterapeutas e 375 Terapeutas Ocupacionais ${ }^{10}$. Tabela 3 - Região de Atuação ( $n=161)$.

\begin{tabular}{lcc}
\hline Variável & Frequência (n) & Percentual (\%) \\
\hline Região & & \\
Metropolitana & 62 & $42,3 \%$ \\
Central & 28 & $16,3 \%$ \\
Não especificado & 25 & $14,3 \%$ \\
Noroeste & 15 & $9,1 \%$ \\
Sudoeste & 11 & $7,4 \%$ \\
Sudeste & 10 & $5,3 \%$ \\
Nordeste & 10 & $5,3 \%$ \\
\hline
\end{tabular}

Fonte: elaborada pelas autoras.

Observar que a maioria dos profissionais atuam em equipe multiprofissional (Figura 1). A partir dos dados analisados, percebe-se a carência de atuação interprofissional, esta pode ser reflexo das lacunas e fragilidades ligadas ao processo de formação do profissional. 
Figura 1 - Atuação em equipe $(n=161)$.

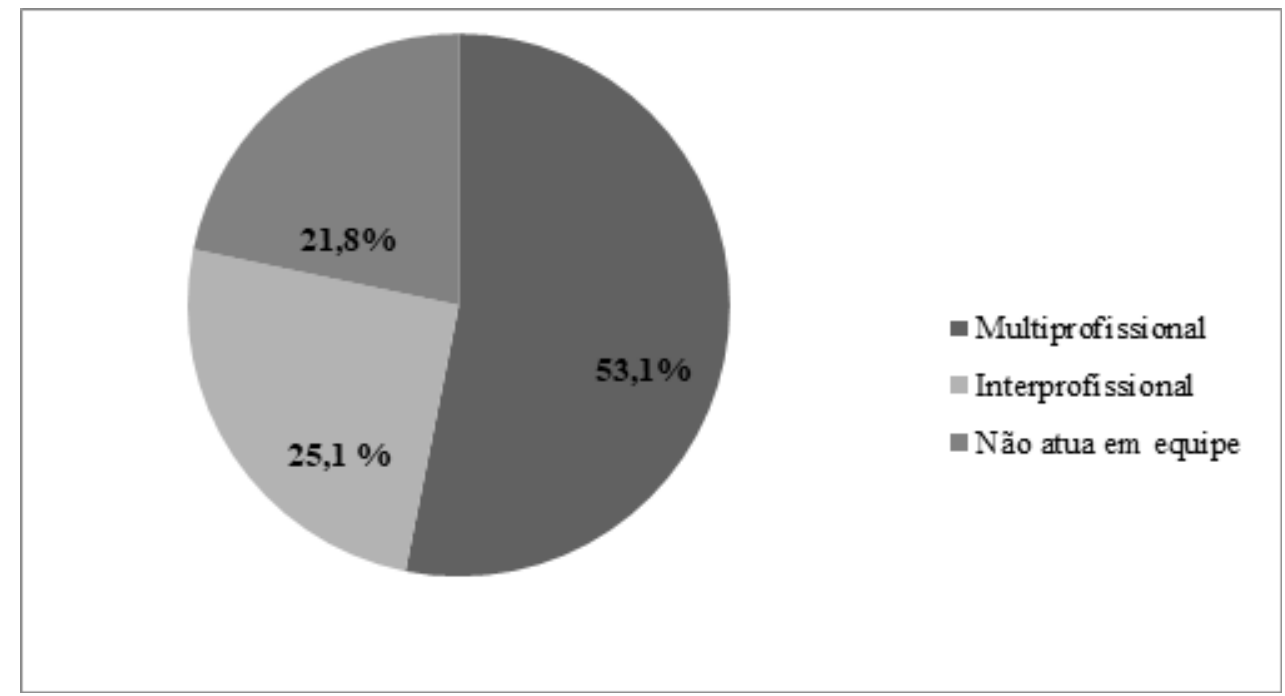

Fonte: elaborada pelas autoras.

Esta realidade vivenciada pelos futuros profissionais de saúde, em algumas Universidade e Instituições Formadoras, apresenta-se em desacordo com o ideário do Sistema Único de Saúde - SUS. Diante disto, estas instituições têm buscado mudar em seu processo de formação, para que assim, por futuros profissionais compreendam o conceito de cuidado integral em saúde e para suprir as necessidades do SUS ${ }^{14}$. Segundo Chiesa et al., ${ }^{15}$ (p.240)

Para que a integração teoria-prática aconteça de maneira efetiva, é necessário que todos os envolvidos na formação (docentes, alunos e profissionais do serviço) entendam e vivam a horizontalização dos saberes, cada um desenvolvendo seus trabalhos de modo a articular conhecimentos complementares, desenvolver as habilidades técnicas e políticas, visando atender as necessidades da população, sempre refletindo sobre suas práticas e avaliando todo o processo.

Assim, para que o trabalho em equipe se torne realidade necessita-se de uma ampla reforma do modelo de formação profissional e de atenção à saúde, esta é discutida em publicações da Organização Mundial da Saúde ${ }^{16}$ e Frenck et al., ${ }^{17}$. Essas e outras transformações que envolvem o processo de formação e de trabalho estão imbuídas no intuito de que o profissional esteja cada vez mais implicado em uma formação crítica, capaz de produzir significado, de trabalhar em equipe, de levar em conta a realidade social, para que assim os futuros profissionais prestem atenção humana e de qualidade ${ }^{14}$. 
Esta transformação no processo de formação é expressa na Resolução nº 569 de 08 de dezembro de 2017, esta ressalta em seu Art. $3^{\circ}$ - Inciso V - que as Diretrizes Curriculares Nacionais - DCN,

\begin{abstract}
devem expressar a formação de um profissional apto a atuar para a integralidade da atenção à saúde, por meio do efetivo trabalho em equipe, numa perspectiva colaborativa e interprofissional. 0 preceito da integralidade aponta também para a interdisciplinaridade - enquanto integração de diferentes campos de conhecimentos; para a interprofissionalidade - ocasião em que há intensa interação entre diferentes núcleos profissionais; e para a intersetorialidade - envolvimento de diferentes setores da sociedade no atendimento das complexas e dinâmicas necessidades de saúde. A integralidade, sustentada por essas premissas, demanda em sua essência (re)situar os usuários na centralidade do processo de produção dos serviços de saúde (BRASIL, 2017, p. s/p) ${ }^{18}$.
\end{abstract}

Neste sentido, as disciplinas compartilhadas pelos cursos da área da saúde poderiam ser otimizadas, refletindo na ampliação da possibilidade de favorecer a convivência e o trabalho em equipe multi/interprofissional, considerados fatores indispensáveis para o alcance da integralidade da atenção. Para Merhy e Franco ${ }^{19}$ (p. 155),

\footnotetext{
"a integralidade é uma diretriz que se organiza acionada por certos projetos terapêuticos que requisitam recursos para a assistência aos usuários, e aí forma-se o encontro entre o mundo das necessidades com os agenciamentos coletivos que operam sobre os trabalhadores certos modos de produzir o cuidado".
}

Pinheiro ${ }^{20}$, ressalta que do ponto de vista jurídico-institucional, a integralidade é definida como um conjunto articulado de ações e serviços de saúde, preventivos e curativos, individuais e coletivos nos diversos níveis de complexidade do sistema. Assim integralidade do cuidado só pode ser obtida em rede. Deve haver algum grau de integralidade "focalizada", mesmo que não seja suficiente, quando uma equipe, em um serviço de saúde, por meio de uma boa articulação de suas práticas, consegue escutar e atender, da melhor forma possível, as necessidades de saúde ${ }^{21}$.

Frente as discussões apresentadas nesta categoria, compreendem-se com maior clareza a realidade sobre a atuação de profissionais de Fisioterapia e Terapia Ocupacional em equipe multi/interprofissional no Estado do Rio Grande do Sul, possibilitando assim, maiores discussões sobre o processo de formação e de trabalho nestes profissionais no Estado. 


\section{Categoria 2 - Atuação em equipe}

O aumento da expectativa de vida, as condições crônicas em saúde e as mudanças do perfil epidemiológico da população, têm exigido das equipes de atenção em saúde acompanhamento prolongado e abordagens de cuidado integral que contemple as múltiplas dimensões das necessidades de saúde. Assim, o trabalho em equipe vem sendo muito debatido em todas as áreas de formação em saúde, este se constitui um tema emergente no campo da saúde em nível global ${ }^{17}$.

Mas, mesmo sendo uma temática recente e que tem sido discutido nos meios acadêmicos, observa-se que em algumas Universidades onde são ofertados os cursos de Fisioterapia e Terapia Ocupacional as discussões limitam-se, em alguns casos, somente ao conceito de atuação interprofissional e multiprofissional em discussões teóricas de disciplinas, quando estas são compartilhadas. Mas estas ainda não são suficientes para que os discentes vivenciem a prática destes conceitos durante o processo de formação, deste modo, a falta de acesso a estas experiências poderá gerar carência de experimentações práticas no decorrer deste, resultando em algumas dificuldades em construir articulações profissionais e trocas de conhecimento e de práticas em equipe.

Nas falas abaixo, observa-se que existe uma dificuldade por parte dos profissionais em compreender o significado do termo multiprofissional, conseguir diferencia-lo do termo interprofissional e aplica-lo na sua prática de atuação.

"A atuação em equipe é maravilhosa, desde que todos entendam o que significa multiprofissionalidade!" [...] (resposta 8).

"A equipe trabalha em um mesmo local, ainda, não tem o sentido de trabalho interdisciplinar" (resposta 50).

"O trabalho em equipe deve ser uma via de mão dupla, onde as trocas deveriam ser feita pelos profissionais, sem medo de perder o paciente ou o conhecimento" (resposta 10).

"[...] quando a atuação em equipe é EFETIVADA, ela sempre apresenta muitas POTENCIALIDADES [..." (resposta 91).

Percebe-se a "tendência dos profissionais de cada área trabalhar de forma isolada e independente das demais" $\left(\right.$ p. 978) ${ }^{22}$. Outra problemática do trabalho em equipe identificada nas respostas dos profissionais 9 e 23 é a hierarquia que parece existir entre a equipe, onde compromete a troca de conhecimentos e a qualidade dos atendimentos multi e interprofissionais. 
"O grande problema pontua apenas na relação com os médicos, que poderia ser mais centrada na equipe do que no individualismo" [...] (resposta 9).

[...] "Possui hierarquia, onde poucas vezes os profissionais não médicos, tem sua opinião respeitada e aceita" (resposta 23).

Para Almeida, Feuerwerker, Llanos ${ }^{23}$ a formação de profissionais em saúde no Brasil é majoritariamente uniprofissional, esta organiza-se a partir de um modelo de disciplinas e em uma racionalidade biomédica, com ênfase na dimensão biológica e fundamentada no anatomopatológico do processo saúde-doença. Esta realidade é reflexo de uma cultura onde a rede de serviços, a gestão e a atenção em saúde são focadas na intervenção do profissional médico e que tem os demais profissionais atuando como auxiliares destes ${ }^{24}$.

Assim, este processo educativo e prático que prioriza os saberes técnicos-científicos e que muitas vezes não estimula a discussão interdisciplinar (comunicativa/integrativa) resulta em uma fragmentação do cuidado22. Frente a esta realidade, o profissional médico ocupa a posição de poder na equipe de saúde, de acordo com Villa, et al., ${ }^{25} 0$ conhecimento específico dos médicos garante, tradicionalmente, legitimidade para exercer o controle sobre os demais membros da equipe e usuários.

Torna-se relevante compreender as relações de poder-saber que podem estar presentes no ambiente e nas relações de traาbalho, visando a uma maior integração entre os trabalhadores que permita a realizaาção de uma assistência de melhor qualidade ${ }^{25}$, pois as relações hierárquicas e as formas de trabalho verticais não beneficiam e ainda prejudicam a qualidade do serviço adoecendo a equipe, tornando-a fragilizada e consequentemente influência na qualidade do trabalho em conjunto.

\section{Categoria 3 - Atuação em equipe e o sujeito}

A origem do trabalho em equipe cujo compromisso é a geração de dispositivos renovados para o trabalho, sendo necessário que cada profissional se familiarize com as outras áreas, de modo legitimado e em relações horizontais. Requer humildade e disponibilidade, num movimento de reconhecimento de dificuldades insolúveis e de posições diferentes em relação a um mesmo objeto ${ }^{26}$.

A atuação em equipe é de grande importância tanto para os profissionais quanto para os usuários, segundo Pinheiro ${ }^{20}$, a integralidade existe em ato e pode ser demandada na organização de serviços e na renovação das práticas de saúde, sendo reconhecida nas práticas que valorizam o cuidado, e que tem em suas concepções a ideia-força de 
considerar o usuário como sujeito a ser atendido e respeitado em suas demandas e necessidades ${ }^{27}$. Como afirmam as falas abaixo:

\author{
"Acredito que uma abordagem de cuidado efetivo se dá mediante uma equipe \\ inter e multidisciplinar a fim de olhar o sujeito em sua integralidade[...]" \\ (resposta 11).
}

"Eu acho fundamental para o atendimento de forma humanizada e resolutiva, sem trabalho em equipe o indivíduo acaba sendo tratado como um quebra cabeça com peças em falta, [...]" (resposta 70).

"[...] Muitas vezes as ações interdisciplinares fazem a diferença no projeto terapêutico, no vínculo, escuta e no repensar de intervenções. O olhar multiprofissional possibilita trocas que trazem beneficios para os pacientes atendidos, e para cada profissional envolvido no processo, capacitando-nos coletivamente" (resposta 92).

Pode-se verificar a necessidade dos profissionais reverem seu entendimento sobre os conceitos multi e interprofissional, passando a deixar de serem vistos como uma forma de divisão de responsabilidades a fim de ser um processo contínuo que permita a ver o usuário como um todo, possibilitando a criação de vínculo e minimizar riscos, pois somente a partir destes, será possível construir junto com equipe e usuários um trabalho de forma horizontal.

\title{
CONSIDERAÇÕES FINAIS
}

Foi possível identificar a falta de adesão dos profissionais, visto que foram obtidas somente 161 respostas do total de 14.269 profissionais cadastrados no CREFITO-5. De acordo com as informações levantadas, certamente um novo modelo de assistência à saúde, fundamentado nos princípios e diretrizes do SUS, requer profissionais com competência técnica e ética, capazes de compreender que, ao acompanhamento em equipe há uma relação de reciprocidade, de interação que pode ajudar no diálogo entre diferentes contextos, desde que haja uma intersubjetividade presente nos sujeitos.

Para tanto, mostra-se necessário um ensino em saúde que propicie a formação de profissionais comprometidos com a integralidade, atores ativos no processo de melhoria da resolutividade do sistema de saúde 


\section{REFERÊNCIAS}

1. OMS. Constituição da Organização Mundial da Saúde (OMS/WHO). 1946. Disponível em: <http://www. direitoshumanos.usp.br/index.php/OMS-Organiza\%C3\%A7\%C3\%A3o-Mundial-da-Sa\%C3\%BAde/constituicao-da-organizacao-mundial-da-saude-omswho.html>. Acessado em: 06 ago. 2020.

2. Capazzolo AA, Casetto SJ, Imbrizi JM, Henz AO, Kinoshita RT, Queiroz MFF. Narrativas na formação comum de profissionais de saúde. Trab. Educ. Saúde. 2014; 12(2):443-56.

3. Ferigollo JP, Kessler TM. Fisioterapia, fonoaudiologia e terapia ocupacional - prática interdisciplinar nos distúrbios da comunicação humana. Rev. CEFAC. 2017; 19(2):147-58.

4. Munari DB, Mota KAMB. Um olhar para a dinâmica do coordenador de grupos. Rev. Eletron. Enferm. 2006; 8(1):150-61.

5. Sousa MF, Hamann, EM. Programa Saúde da Família no Brasil: uma agenda incompleta? Ciên. Saúde Colet. 2009; 14(Supl1):1325-35.

6. Robins H, Finley M. Por que as equipes não funcionam. Rio de Janeiro: Editora Campus, 1997.

7. Peduzzi M. Equipe multiprofissional de saúde: conceito e tipologia. Rev. Saúde Púb. 2001; 35(1):103-109.

8. Araújo TAM, Vasconcelos ACCP, Pessoa TRRF, Forte FDS. Multiprofissionalidade e interprofissionalidade em uma residência hospitalar: o olhar de residentes e preceptores. Interface: Comunic., Saúde e Educ. 2017; 21(62):601-13.

9. Bardin, L. Análise de Conteúdo. Lisboa, Portugal; Edições 70, LDA, 2009.

10. Conselho Regional de Fisioterapia e Terapia Ocupacional - CREFITO-5. Por tipo de registro - 2018. Disponível em: <http://crefito5.org.br/servicos-online-estatistica-registrados>. Acessado em 23 jun. 2018. 
11. Silva EB. A formação de profissionais no cuidado terapêutico de sujeitos com lesões neurológicas. Dissertação (Mestrado em Distúrbios da Comunicação Humana) - Universidade Federal de Santa Maria - UFSM, 2016.

12. Ribeiro RP, Marziale MHP, Martins JT, Galdino MJQ, Ribeiro PHV. Estresse ocupacional entre trabalhadores de saúde de um hospital universitário. Rev. Gaúcha Enferm. 2018;39:e65127.

13. Kolankiewicz ACB, Schmidt CR, Carvalho REFL, Spies J, Dal Pai S, Lorenzini E. Cultura de segurança do paciente na perspectiva de todos os trabalhadores de um hospital geral. Rev. Gaúcha Enferm. 2020;41:e20190177.

14. Ceccim RB, Feuerwerker LCM. Mudanças na graduação das profissões de saúde sob o eixo da integralidade. Cad. Saúde Púb. 2004; 20(5):1400-10.

15. Chiesa AM, Nascimento DDG, Braccialli LAD, Oliveira MAC. A Formação de Profissionais da Saúde: Aprendizagem Significativa à Luz da Promoção da Saúde. Cogitare Enferm. 2007; 12(2):236-40.

16. World Health Organization (WHO). Framework for action on interprofessional education and collaborative practice. Geneva: WHO; 2010

17. Frenck J, Chen L, Bhutta ZA, Cohen J, Crisp N, Evans T, Fineberg H, Garcia P, Ke Y, Kelley P, Kistnasamy B, Meleis A, Naylor D, Pablos-Mendez A, Reddy S, Scrimshaw S, Sepulveda J, Serwadda D, Zurayk H. Health professionals for a new century: transforming education to strengthen health systems in an interdependent world. Lancet, 2010; 376(9756): 1923-57.

18. Brasil. Plenário do Conselho Nacional de Saúde (CNS). Resolução nº 569 de 8 de dezembro de 2017. Disponível em: <http://conselho.saude.gov.br/resolucoes/2017/Res0569.pdf>. Acesso em: 16 jul. 2018.

19. Merhy EE, Franco TB. Cartografias do trabalho e cuidado em saúde. Tempus Actas Saude Colet. 2012; 6(2):151-63.

20. Pinheiro R. Integralidade em saúde. Dicionário da Educação Profissional em Saúde. 2.ed. Rio de Janeiro: EPSJV, 2008. 
21. Cecílio LCO, Merhy EE. A integralidade do cuidado como eixo da gestão hospitalar. Campinas: Unicamp, 2003. Disponível em: <http://www.hmdcc.com.br/wp-content/uploads/2018/04/Cecilio-A-INTEGRALIDADE-DO-CUIDADO-COMO-EIXO-DA-GEST\%C3\%830-HOSPITALAR.pdf>. Acessado em 16 jul. 2018.

22. Peduzzi M, Norman IJ, Germani ACCG, Silva JAM, Souza GC. Educação interprofissional: formação de profissionais de saúde para o trabalho em equipe com foco nos usuários. Rev Esc Enferm USP. 2013; 47(4):977-83.

23. Almeida MJ, Feuerwerker L, Llanos M. A educação dos profissionais de saúde na América Latina: teoria e prática de um movimento de mudança. São Paulo: Hucitec; 1999.

24. Ribeiro EM, Pires D, Blank VL. A teorização sobre processo de trabalho em saúde como instrumental para análise do trabalho no Programa Saúde da Família. Cad. Saúde Pública, 2004; 20(2):438-46.

25. Villa EA, Aranha AVS, Silva, LLT, Flôr CR. As relações de poder no trabalho da Estratégia Saúde da Família. Saúde Debate, 2015; 39(107):1044-52.

26. Tribarry IN. Aproximações sobre a transdisciplinaridade: algumas linhas históricas, fundamentos e princípios aplicados ao trabalho de equipe. Psicol Refl Crit., 2003; 16(3):483-90.

27. Silva JPV, Pinheiro R, Machado FRS. Necessidades, demanda e oferta: algumas contribuições sobre os sentidos, significados e valores na construção da integralidade na reforma do setor saúde. Saúde em Debate, 2003; 27(65):234-42. 